capacity of one laboratory. Kool is very aware of this and emphasizes that he currently has many ongoing collaborations and is happy to embark on new ones. Ultimately he sees the future of this library in the commercial market, where the end users determine the properties they need and the compounds that detect these properties by shifting color are made to order.
Although this is not yet a reality, the day when a change in color will easily answer important biological questions may be only around a few corners.

\section{Nicole Rusk}

\section{RESEARCH PAPER}

Gao, J. et al. Modified DNA Analogues that sense light exposure with color changes. J. Am. Chem. Soc. published online 18 September 2004.

\title{
FLUORESCENT VIRUS LETS RESEARCHERS IN ON THE GAG
}

A new variant of HIV with a fluorescent tag inserted into the coding sequence of Gag, the virus' major structural protein, offers a valuable new tool for visualization of the processes of viral-cell interaction, entry and release.

Early efforts to generate fluorescently labeled HIV have succeeded at revealing key steps of viral entry, localization and replication, although none of these strains appears to retain full functionality. Several strategies have focused on applying tags to Gag, the primary structural protein of HIV. Gag consists of multiple functional domains that are processed post-translationally by HIV protease-mediated cleavage; as such, any manipulation must be made with care not to disrupt this processing. Several groups have successfully tagged the $C$ terminus of the full-length Gag protein, although the resulting labeled virus has typically been noninfectious.

Hans-Georg Kräusslich and his colleagues at the Universitätsklinikum Heidelberg and Deutches KrebsForschungszentrum (Heidelberg, Germany) sought to develop a GFP-labeled virus capable of proper assembly, infection and release from host cells, and found elements of the structure of unprocessed Gag that seemed to provide the opportunity they needed. A relatively unstructured region between the globular matrix (MA) and capsid (CA) domains seemed likely to tolerate modification, and indeed, the insertion of a 12-residue c-myc epitope tag at the $\mathrm{C}$ terminus of MA appeared to have no detrimental effects on Gag processing or the assembly of functional virions. Moreover, the epitope tag was detectable by immunostaining of infected cells.

As a next step, the investigators replaced c-myc with the considerably larger coding region for enhanced green fluorescent protein (EGFP). Transfection of this modified HIV plasmid into HeLa cells resulted in strong fluorescence from within syncytia, and MA-EGFP was readily detectable by western blotting. Mature virions were also clearly visible by electron microscopy, although elevated accumulation at the plasma membrane suggested some reduction in budding and release relative to wild-type virus. The released Gag-EGFP virions were infectious but, again, at a reduced level. Kräusslich and his colleagues are currently investigating these replicative defects, but found that the deficits were overcome to some extent by cotransfection of cells with a plasmid encoding wild-type virus, which led to the production of hybrid, labeled virions.

Kräusslich foresees widespread use of this Gag-EGFP virus as a tool for studying HIV entry and release from host cells, and as a valuable reagent for the development of new drugs. "Specifically, one may look at individual steps in different cell lines and primary cells and use the virus to visualize the action of antiviral compounds," he says. "We are also using a modified version of this construct for studying virus release in a quantitative manner by directly determining fluorescence in the culture medium - in the future, this assay will be used for screening of compounds inhibiting HIV release."

\section{Michael Eisenstein}

\section{RESEARCH PAPERS}

Müller, B. et al. Construction and characterization of a fluorescently labeled infectious human immunodeficiency virus type 1 derivative. J. Virol. 78, 10803-10813 (2004). 